\title{
Toll-Like Receptor-Mediated Free Radical Generation in Clonorchis sinensis Excretory-Secretory Product-Treated Cholangiocarcinoma Cells
}

\author{
Young Yil Bahk', Jhang Ho Pak ${ }^{2, *}$ \\ 'Department of Biotechnology, Konkuk University, Chungju 27478, Korea; ${ }^{2}$ Department of Convergence Medicine, University of Ulsan College of \\ Medicine and Asan Institute for Life Sciences, Asan Medical Center, Seoul 05505, Korea
}

\begin{abstract}
Clonorchiasis, caused by direct contact with Clonorchis sinensis worms and their excretory-secretory products (ESPs), is associated with chronic inflammation, malignant changes in bile ducts, and even cholangiocarcinogenesis. Our previous report revealed that intracellular free radicals enzymatically generated by $C$. sinensis ESPs cause NF-KBmediated inflammation in human cholangiocarcinoma cells (HuCCT1). Therefore, the present study was conducted to examine the role of upstream Toll-like receptors (TLRs) on the initial host innate immune responses to infection. We found that treatment of HuCCT1 cells with native ESPs induced changes in TLR MRNA levels in a time-dependent manner, concomitant with the generation of free radicals. ESP-mediated free radical generation was markedly attenuated by preincubation of the cells with TLR1-4-neutralizing antibodies, indicating that at least TLR1 through 4 participate in stimulation of the host innate immune responses. These findings indicate that free radicals triggered by ESPs are critically involved in TLR signal transduction. Continuous signaling by this pathway may function in initiating $\mathrm{C}$. sinensis infection-associated inflammation cascades, a detrimental event leading to progression to more severe hepatobiliary diseases.
\end{abstract}

Key words: Clonorchis sinensis, Toll-like receptor (TLR), free radical, excretory-secretory product (ESP), cholangiocarcinoma cell line

Members of the Toll-like receptor (TLR) family are among the most important intra- and extracellular ligand-recognition receptors, which actively function as potent initiators of host innate immune responses and activate proinflammatory signaling cascades $[1,2]$. TLRs are evolutionarily highly conserved molecules. They recognize molecular patterns on pathogenic organisms and anti-pathogenic functions of the host, and assemble the immune responses for effectiveness against specific pathogens. TLR-mediated inflammation is a primary protective host response to ensure exclusion of detrimental threats caused by infectious agents, and promotes healing [3]. TLRs play a central role in the detection of pathogens by recognizing pathogen-associated molecular patterns derived from a variety of pathogens or host-derived, damage-associated molecular patterns [4]. To date, at least 10 members of the TLR family have been identified in humans, although not all have been

\footnotetext{
- Received 14 July 2016, revised 24 August 2016, accepted 25 August 2016.

*Corresponding author (jhpak@amc.seoul.kr)

(C) 2016, Korean Society for Parasitology and Tropical Medicine

This is an Open Access article distributed under the terms of the Creative Commons

Attribution Non-Commercial License (http://creativecommons.org/licenses/by-nc/4.0) which permits unrestricted non-commercial use, distribution, and reproduction in any

medium, provided the original work is properly cited.
}

assigned a specific ligand. Various cells are able to discriminate between infectious pathogens, based on their profiles of TLR and proinflammatory mediators, and differential expression patterns. This enables diverse responses against different types of pathogens [5-7]. However, prolonged inflammation resulting from excessive production of inflammatory cytokines and chemokines via TLR-mediated signaling events can be detrimental owing to host toxicity and tissue damage. Thus, while T-helper 1 (Th1)-based inflammatory consequences orchestrated by TLRs are involved in eliminating pathogenic infections, they may also induce fatal pathogenic consequences [8]. Similarly, the pathogen-modulated Th2-based TLR-mediated signaling pathway develops immune responses beneficial for the pathogen, leading to disease progression.

Clonorchiasis, caused by chronic infection with Clonorchis sinensis, is an undeniable public health problem in endemic regions of Southeast Asian countries. These flukes reside in the peripheral bile ducts of infected individuals for long periods, provoking pathological changes in the bile ducts and surrounding liver tissues, including inflammation, metaplasia of mucin-producing cells in the mucosa, progressive periductal fibrosis, and hyperplasia of epithelial cells. In particular, persis- 


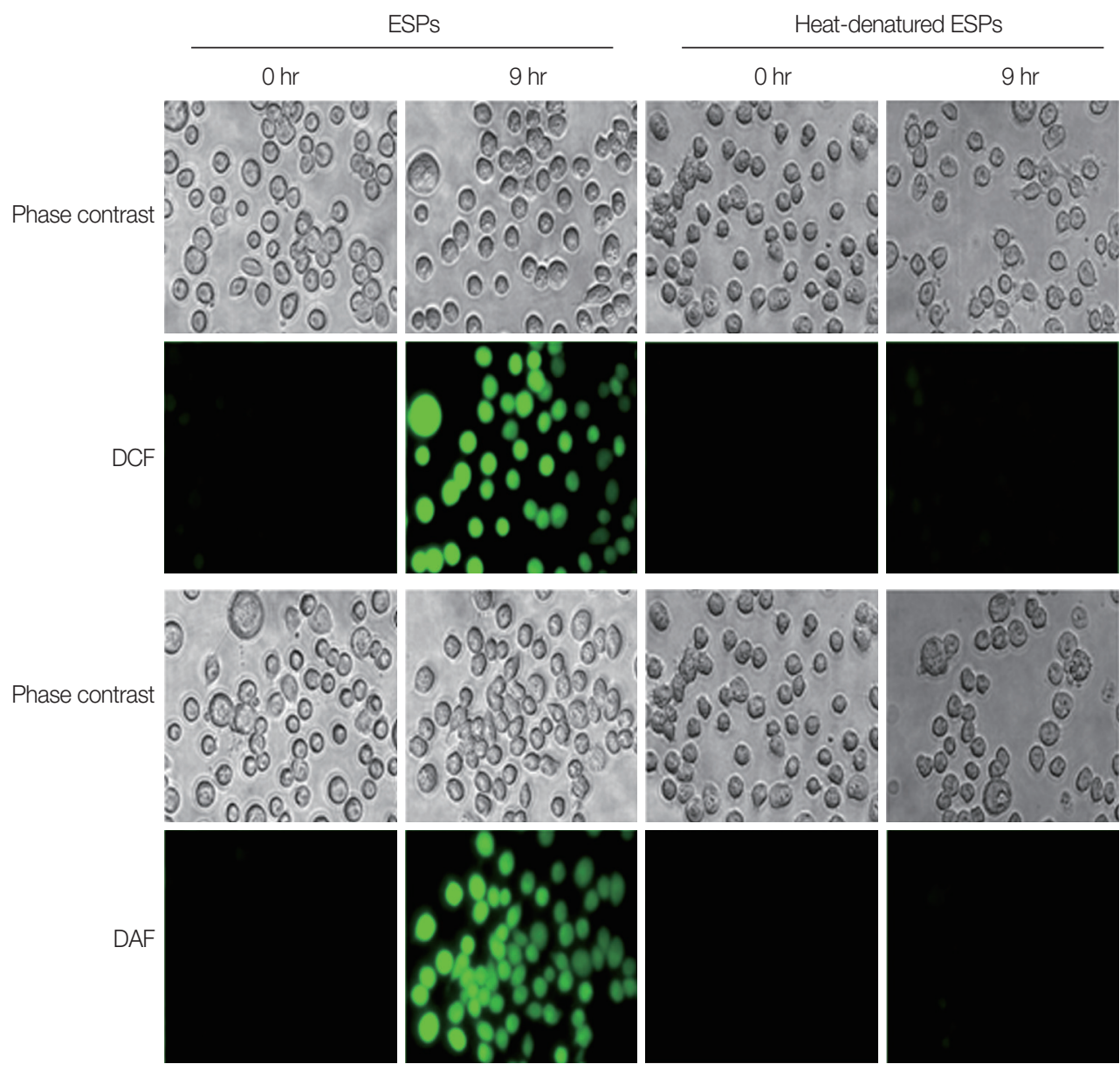

Fig. 1. Intracellular free radical generation in native ESP-treated HuCCT1 cells. Serum-starved cells grown on Aclar plates were incubated in phenol red-free medium containing $800 \mathrm{ng} / \mathrm{ml}$ native or heat-denatured ESPs for $9 \mathrm{hr}$. Cells were then washed with Hank's balanced salt solution (HBSS) and were treated with $10 \mu \mathrm{M}$ of $\mathrm{CM}-\mathrm{H}_{2} \mathrm{DCFDA}$ (for ROS detection) or $5 \mu \mathrm{M}$ of DAF-FM (for RNS detection) for $30 \mathrm{~min}$ at $37^{\circ} \mathrm{C}$ in the dark. After washing with HBSS, cells were fixed, air dried, and inversely applied to slides with mounting solution. Images were captured under a fluorescent microscope equipped with a standard FITC excitation/emission filter. To avoid photooxidation of the fluorescent probes, the fluorescent images were collected with a single rapid scan and identical parameters. Original magnification $\times 100$.

tent injury and inflammation of bile duct epithelia, resulting from both mechanical and chemical irritation, contribute to clonorchiasis-associated cholangiocarcinogenesis. Mechanical damage is caused by physical contact with worms during their feeding and migratory activities, whereas chemical damage is caused by their excretory-secretory products (ESPs). The ESPs are predominantly composed of various proteins, some of which are immune reactive with the sera of $C$. sinensis-infected patients [9]. Cells exposed to live fluke ESPs display diverse pathophysiological responses, including proliferation, apoptosis, and inflammation [10-13].

We previously reported that treatment of HuCCT1 human cholangiocarcinoma (CCA) cells with C. sinensis ESPs results in elevated levels of intracellular free radicals in a dose- and time-dependent manner, due to activation of NADPH oxidase (NOX), xanthine oxidase (XO), and inducible nitric oxide synthase (iNOS). The enzymatic production of free radicals subsequently participates in NF-kB-mediated inflammation $[13,14]$. Since TLRs are known to function as sensors for innate and adaptive immune responses during helminth infection [15], we examined feasible roles of TLRs in the generation of free radicals, a prerequisite step for the NF-kB-mediated inflammatory response to $C$. sinensis ESPs.

C. sinensis ESPs were prepared as described previously [13]. HuCCT1 cells were cultured in RPMI 1640 medium (Life Technologies, Grand Island, New York, USA) supplemented 
Table 1. Primer sequences for amplification of TLR isoforms by RT-PCR

\begin{tabular}{|c|c|c|c|}
\hline Gene name & Direction & Primers & Amplicon size (bp) \\
\hline \multirow[t]{2}{*}{ TLR1 } & Forward & 5' - CGTAAAACTGGAAGCTITGCAAGA -3' & 890 \\
\hline & Reverse & 5'- CCTTGGGCCATTCCAAATAAGTCC -3' & \\
\hline \multirow[t]{2}{*}{ TLR2 } & Forward & 5'- GGCCAGCAAATTACCTGTGTG -3' & 615 \\
\hline & Reverse & 5' - CCAGGTAGATCTTGGTGTTCA -3' & \\
\hline \multirow[t]{2}{*}{ TLR3 } & Forward & 5' - ACATCCCTGAGCTGTCAAGC -3' & 320 \\
\hline & Reverse & 5'- CCGCCTCAAAGTCCCTTTCT -3' & \\
\hline \multirow[t]{2}{*}{ TLR4 } & Forward & 5' - CTGCAATGGATCAAGGACCA -3' & 623 \\
\hline & Reverse & 5'- TCCCACTCCAGGTAAGTGTT -3' & \\
\hline \multirow[t]{2}{*}{ TLR5 } & Forward & 5' CATTGTATGCACTGTCACTC -3' & 486 \\
\hline & Reverse & 5'- CCACCACCATGATGAGAGCA -3' & \\
\hline \multirow[t]{2}{*}{ TLR6 } & Forward & 5'- TAGGTCTCATGACGAAGGAT -3' & 1,108 \\
\hline & Reverse & 5'- GGCCACTGCAAATAACTCCG -3' & \\
\hline \multirow[t]{2}{*}{ TLR7 } & Forward & 5' - AGTGTCTAAAGAACCTGG -3' & 545 \\
\hline & Reverse & 5'- CTTGGCCTTACAGAAATG -3’ & \\
\hline \multirow[t]{2}{*}{ TLR8 } & Forward & 5' CAGAATAGCAGGCGTAACACATCA -3’' & 637 \\
\hline & Reverse & 5' - AATGTCACAGGTGCATTCAAAGGG -3' & \\
\hline \multirow[t]{2}{*}{ TLR9 } & Forward & 5' - TTATGGACTTCCTGCTGGAGGTGC -3’' & -- \\
\hline & Reverse & 5' - CTGCGTITGTCGAAGACCA -3’' & \\
\hline \multirow[t]{2}{*}{ TLR10 } & Forward & 5' - CAATCTAGAGAAGGAAGATGGTTC -3’' & 659 \\
\hline & Reverse & 5'- GCCCTTATAAACTTGTGAAGGTGT -3' & \\
\hline
\end{tabular}
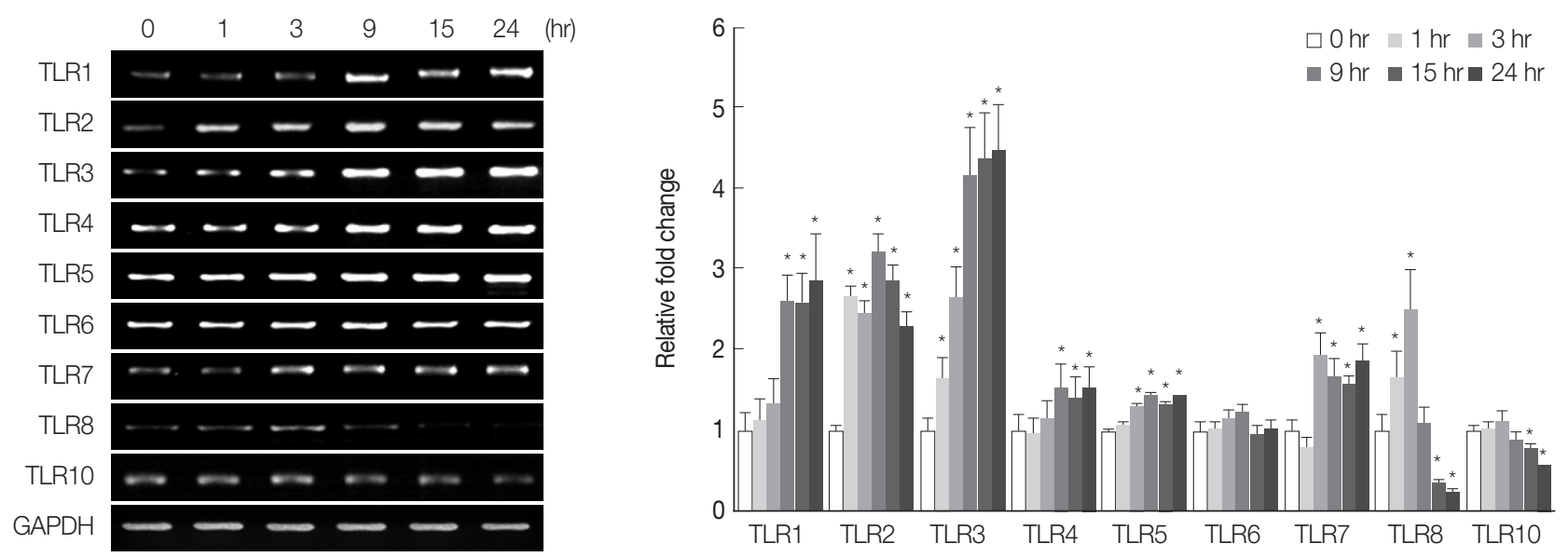

Fig. 2. Effect of ESPs on expression of TLR mRNA isoforms. HuCCT1 cells were treated with 800 ng/ml ESPs, harvested between 0 and $24 \mathrm{hr}$, and subjected to semi-quantitative RT-PCR analysis. Individual data were quantified as densitometric units, and normalized with GAPDH mRNA. Data in the graph are shown as fold changes relative to the $0 \mathrm{hr}$ time point, and presented as means \pm SE of 3 independent experiments ( ${ }^{*} P<0.05$, compared with $0 \mathrm{hr}$ ).

with 10\% FBS and an antibiotic mixture. For CsESP treatment, cells were seeded at -70\% confluence and grown for $24 \mathrm{hr}$ under standard culture conditions. Cells were gradually deprived of serum by incubating in 1\% FBS, followed by incubation in serum-free medium for $3 \mathrm{hr}$. These serum-starved cells were incubated with $800 \mathrm{ng} / \mathrm{mL}$ CsESP for the indicated times.

Heat-denatured ESPs failed to induce the production of intracellular free radicals, as assessed by the detection of fluorescent probes:5-(and-6)-chloromethyl-2', 7' -dihydrodichloro- fluorescein diacetate acetyl ester (CM- $\mathrm{H}_{2}$ DCFDA) for detection of reactive oxygen species (ROS), and 4-amino-5-methylamino-2', $7^{\prime}$-dichlorofluorescein diacetate (DAF-FM) (Molecular Probes, Eugene, Oregon, USA) for detection of reactive nitrogen species (RNS). This result indicates that native protein components in CsESP are responsible for triggering free radical generation in the cells (Fig. 1).

For analysis of TLR mRNA expression, total RNA was extracted from CsESP-treated cells at each time point, using an 


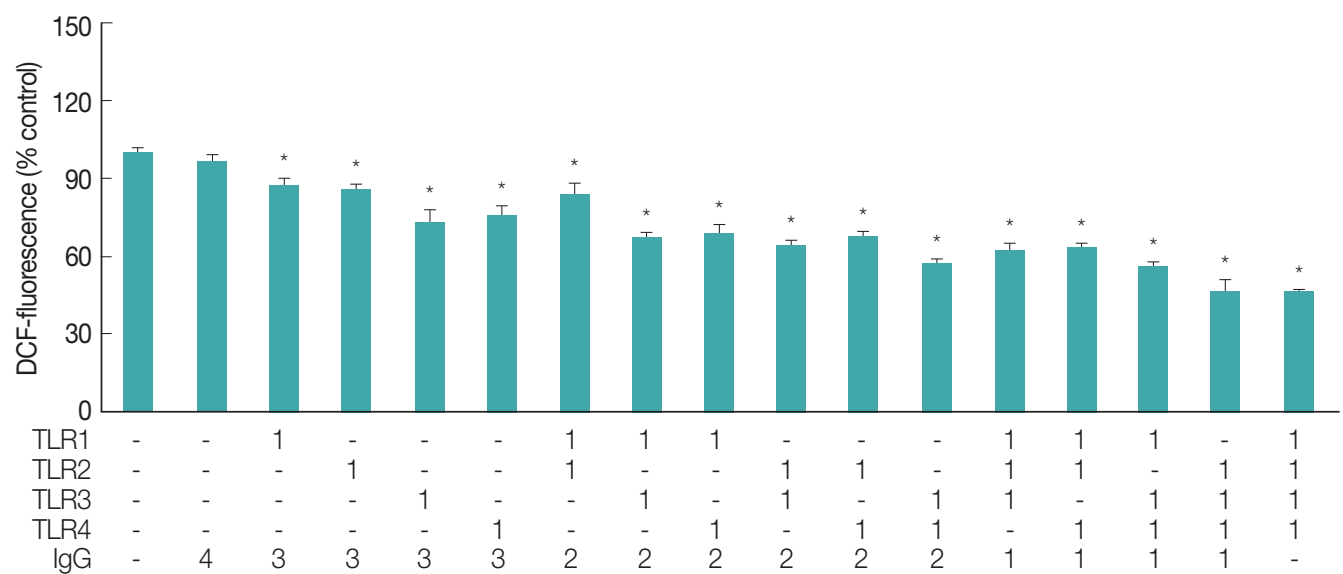

Fig. 3. Inhibitory effect of TLR1, 2, 3, and 4 neutralization on ESP-triggered ROS generation. HuCCT1 cells grown in 48-well plates were preincubated with the indicated single or combined TLR monoclonal antibodies for $2 \mathrm{hr}$, and then treated with $800 \mathrm{ng} / \mathrm{ml}$ CsESP for 9 $\mathrm{hr}$. The total concentration of antibodies in each well was maintained by the addition of non-specific lgG. After incubation with $\mathrm{CM}-\mathrm{H}_{2} \mathrm{D}-$ CFDA, the levels of DCF fluorescence were measured using a spectrofluorometer with excitation and emission wavelengths of 485 and $538 \mathrm{~nm}$, respectively. The value for background fluorescence measured for empty wells was subtracted from all other values. Values were converted to percentages for comparison with control treated with only CsESP. Values are presented as means \pm SE of 3 independent experiments ( ${ }^{*} P<0.05$, compared with the ESP only-treated control).

RNeasy mini kit (Qiagen, Valencia, California, USA), and a semi-quantitative RT-PCR was performed according to the manufacturers' instructions (SuperScript II First-strand, Invitrogen, Carlsbad, California, USA; ExTaq, TaKaRa Bio, Inc., Shiga, Japan). Table 1 depicts the primer pairs used for RT-PCR, which were designed using PrimerDesigner software to target the CDNA sequences for each human TLR isoform in the GeneBank database. The sequences of the primer pair targeting glyceraldehyde-3-phosphate dehyrogenase (GAPDH), used as an internal control, were as follows: $5^{\prime}$-ACCCAGAAGACTGTGGATGG-3' (forward) and 5'-CAGGAATG-AGCTTGACAAAG-3' (reverse). PCR products were separated on $1.0 \%$ agarose gels, and images were captured for quantitation of DNA band densities. We observed that mRNA levels of TLR2, 3, and 5 were relatively abundant compared with those of other TLR isoforms, and TLR9 was not detectable (data not shown). Consistent with this finding, a SV40-transformed normal human cholangiocyte cell line (H69) has been shown to express all the known TLR (1-10) mRNAs to different amounts [16]. The absence of TLR9 in HuCCT1 cells was probably due to the specific cell-type. The differences between the different cell types may be attributed to their classification as cancerous versus non-cancerous bile duct epithelial cells. The CsESP treatment resulted in elevated levels of TLR1, 2, 3, 4, 5, and 7 transcripts in a time-dependent manner. However, no change was evident in TLR6 mRNA expression during the entire period of
ESP exposure (Fig. 2). Significant increases in levels of TLR2 and 3 were observed as early as $1 \mathrm{hr}$, TLR5 and 7 after $3 \mathrm{hr}$, and TLR1 and 4 after 9 hr of ESP exposure. Elevated expression was maintained for up to $24 \mathrm{hr}$ of ESP exposure. The expression of TLR8 mRNA increased at $1 \mathrm{hr}$, reached a maximal level at $3 \mathrm{hr}$, and then dramatically decreased to a level below that of an untreated control between 15 and $24 \mathrm{hr}$ of exposure. The level of TLR10 mRNA expression gradually declined between 15 and $24 \mathrm{hr}$ of ESP exposure. The differential biphasic patterns of TLR isoform mRNA expression in response to ESPs remains to be elucidated. Although various pathogens typically cause induction of TLR expression, down-regulation of TLR expression seems to be a critical strategy for the success of specific pathogens [17]. These findings suggest that $C$. sinensis ESPs may be rich in agonists for several members of the TLR family, thus contributing to various host innate immune responses. It has been also reported that expression of TLR2 and 4 is induced by ESPs from Opisthorchis viverrini $[18,19]$. Since TLRs recognize pathogen-associated molecular patterns that are expressed on infectious agents, host cells may recognize liver fluke ESPs through differential activation of TLRs, leading to either the development of effective immunity or pathologic progression, such as activation of the innate mucosal inflammation cascade. In the mouse model for clonorchiasis, the expression of TLR2 and 4 mRNA and proteins in infected livers is up-regulated relatively early in infection (day 28 post-infec- 
tion), indicating that both TLR2 and TLR4 participate in the stimulation of the innate immune response during $C$. sinensis infection [20].

To take into accounts the involvement of TLRs in free radical generation, ESP-induced TLR activation was blocked using functional grade monoclonal antibodies against TLR1, 2, 3, or 4 (Affymetrix-eBioscience, San Diego, California, USA), followed by a DCF fluorescence assay. The TLR1 through 4 antibodies were employed for this purpose, since they are the only ones functionally proven and commercially available to our knowledge. As shown in Fig. 3, blockage of individual TLRs resulted in approximately $12-27 \%$ reduction in the ROS generation triggered by ESPs. This reduction effect was further enhanced when anti-TLR antibodies were added in combination. Reduction rates were proportional to the number of TLR members targeted by the antibodies (addition of 2 combined antibodies resulted in $17-43 \%$ reduction in ROS levels, and addition of 3 antibodies resulted in 36-54\% reduction). In particular, pretreatment with a combination of antibodies (TLR2, 3, and 4, or TLR1, 2, 3, and 4) suppressed ROS generation by $-55 \%$ relative to that triggered by ESP treatment alone, indicating a synergistic inhibitory effect. As assessed by a DAF fluorescence assay, RNS levels also decreased in proportion to the number of anti-TLR antibodies combined in the samples (data not shown). These results suggest that free radical generation in HuCCT1 cells exposed to C. sinensis ESPs may be partially mediated by the activation of various members of the TLR family, which is a prerequisite for signaling downstream NF-kB-induced inflammation.

Endogenous free radicals originate from multiple sources, including the electron transport chain in mitochondria and enzymatic reactions involving NOX, XO, NOS, lipoxygenases, and cyclooxygenases. Depending on the levels generated, free radicals may cause serious cellular injury and contribute to the pathogenesis of several diseases. They may also act as secondary messengers for signal transduction and gene regulation in a variety of cell types under diverse biological conditions. During inflammation and immune responses, activated phagocytic cells generate a free radical-dependent respiratory burst that directs toxicity toward invading pathogens. In addition to their antiseptic functions, release of free radicals by damaged tissues recruits inflammatory cells to the sites of injury, thus demonstrating that free radicals can orchestrate inflammatory responses [21]. Increasing lines of evidence indicate that ROSgenerating NOXs in non-phagocytic cells function as key play- ers in TLR-mediated intracellular cascades, which activate redox-dependent transcription factors, such as NF- $\mathrm{kB}$ and AP-1, as well as cytokine production following a variety of inflammatory stimuli [7]. Therefore, it is tempting to speculate that $C$. sinensis ESPs activate the host inflammatory signaling cascade, partially through TLRs. Disruption of host cellular redox homeostasis and prolonged endogenous crosstalk between free radicals and TLR activation exacerbate a chronic inflammatory environment in the bile duct epithelium and surrounding liver tissues, eventually leading to the development of more severe hepatobiliary abnormalities. Further examination of TLR-mediated pathophysiologic processes in response to CsESP, including their regulation and the interaction between TLRs, is necessary to elucidate the mechanisms underlying the progression and cause of clonorchiasis-associated disorders.

\section{ACKNOWLEDGMENT}

This work was supported by a grant from the National Research Foundation of Korea (NRF) funded by the Korea Government (MEST) (NO. 2012R1A2A2A01014237).

\section{CONFLICT OF INTEREST}

We have no conflict of interest related to this study.

\section{REFERENCES}

1. Akira S, Takeda K. Toll-like receptor signaling. Nat Rev Immunol 2004; 4: 499-511.

2. Kawai T, Akira S. Toll-like receptors and their crosstalk with other innate receptors in infection and immunity. Immunity 2011; 34: 637650.

3. Zeytun A, Chaudhary A, Pardington P, Cary R, Gupta G. Induction of cytokines and chemokines by Toll-like receptor signaling: strategies for control of inflammation. Crit Rev Immunol 2010; 30: 53-67.

4. Akira S, Uematsu S, Takeuchi O. Pathogen recognition and innate immunity. Cell 2006; 124: 783-801.

5. Junpee A, Tencomnao T, Sanprasert V, Nuchprayoon S. Association between Toll-like receptor 2 (TLR2) polymorphisms and asymptomatic bancroftian filiariasis. Parasitol Res 2010; 107: 807-816.

6. Hart BE, Tapping RI. Genetic diversity of Toll-like receptors and immunity to M. leprae infection. J Trop Med 2012; 2012: 415057.

7. Ogier-Denis E, Mkaddem SB, Vandewalle A. NOX enzymes and Toll-like receptor signaling. Semin Immunopathol 2008; 30: 291300.

8. Liu Q, Ding JL. The molecular mechanisms of TLR-signaling coop- 
eration in cytokine regulation. Immunol Cell Biol 2016; 94: 538542.

9. Pak JH, Kim DW, Moon JH, Nam JH, Kim JH,Ju JW, Kim TS,SeoSB. Differential gene expression profiling in human cholangiocarcinoma cells treated with Clonorchis sinensis excretory-secretory products. Parasitol Res 2009; 104: 1035-1046.

10. Thuwajit C, Thuwajit $P$, Kaewkes S, Sripa B, Uchida K, Miwa M, Wongkham $\mathrm{S}$. Increased cell proliferation of mouse fibroblast NIH-3T3 in vitro induced by excretory/secretory products from Opisthorchis viverrini. Parasitology 2004; 129: 455-464.

11. Kim YJ, Choi MH, Hong ST, Bae YM. Proliferative effects of excretory/secretory products from Clonorchis sinensis on the human epithelial cell line HEK293 via regulation of the transcription factor E2F1. Parasitol Res 2008; 102: 411-417.

12. Serradell MC,Guasconi L,Cervi L,Chiapello LS,Masih DT. Excretory-secretory products from Fasciola hepatica induce eosinophil apoptosis by a caspase-dependent mechanism. Vet Immunol Immunopathol 2007;117: 197-208.

13. Nam JH, Moon JH, Kim IK, Lee MR, Hong SJ, Ahn JH, Chung JW, Pak JH. Free radicals enzymatically triggered by Clonorchis sinensis excretory-secretory products cause NF- $\mathrm{kB}$-mediated inflammation in human cholangiocarcinoma cells. Int J Parasitol 2012; 42: 103-113.

14. Pak JH, Moon JH, Hwang SJ, Cho SH, Seo SB, Kim TS. Proteomic analysis of differentially expressed proteins in human cholangiocarcinoma cells treated with Clonorchis sinensis excretory-secretory products. J Cell Biochem 2009; 108: 1376-1388.

15. Venugopal PG, Nutman TB, Semnani RT. Activation and regulation of Toll-like receptors (TLRs) by helminth parasites. Immunol Res 2009; 43: 252-263.

16. Chen XM, O’Hara SP, LaRusso NF. The immunobilogy of cholangiocytes. Immunol Cell Biol 2008; 86: 497-505.

17. Alvarez JI. Inhibition of Toll like receptor immune responses by microbial pathogens. Front Biosci 2005; 10: 582-587.

18. Pinlaor S, Tada-Oikawa S, Hiraku Y, Pinlaor P, Ma N, Sithithaworn $\mathrm{P}$, Kawanishi S. Opisthorchis viverrini antigen induces the expression of Toll-like receptor 2 in macrophage RAW cell line. Int J Parasitol 2005; 35: 591-596.

19. Ninlawan K, O’Hara ST, Splinter PL, Yonggvanit P, Kaewkes S, Surapaitoon A, LaRusso, NF, Sripa B. Opisthorchis viverrini excretory/secretory products induce toll-like receptor 4 upregulation and production of interleukin 6 and 8 in cholangiocytes. Parasitol Int 2010; 59: 616-621.

20. Yan C, Li XY, Li B, Zhang BB, Xu JT, Hua H, Yu Q, Liu ZZ, Fu LL, Tang RX, Zheng KY. Expression of Toll-like receptor (TLR) 2 and TLR4 in the livers of mice infected by Clonorchis sinensis. J Infect Dev Ctries 2015; 9: 1147-1155.

21. Martinon F. Signaling by ROS drives inflammasome activation. Eur J Immunol 2010; 40: 616-619. 